\title{
MRI-BASED MORPHOMETRIC ANALYSis OF Typical and Atypical Brain DeVelopment
}

\author{
David N. Kennedy ${ }^{1,2 *}$, Christian Haselgrove ${ }^{1}$, and Sean McInerney ${ }^{1}$ \\ ${ }^{1}$ Center for Morphometric Analysis, Department of Neurology, Massachusetts General Hospital, Boston, Massachusetts \\ ${ }^{2} \mathrm{MGH} / \mathrm{MIT} / \mathrm{HMS}$ Athinoula A. Martinos Center for Biomedical Imaging, Boston, Massachusetts
}

The neuroinformatics landscape in which human brain morphometry occurs has advanced dramatically over the past few years. Rapid advancement in image acquisition methods, image analysis tools and interpretation of morphometric results make the study of in vivo anatomic analysis both challenging and rewarding. This has revolutionized our expectations for current and future diagnostic and investigative work with the developing brain. This paper will briefly cover the methods of morphometric analysis that available for neuroanatomic analysis, and tour some sample results from a prototype retrospective database of neuroanatomic volumetric information. From these observations, issues regarding the anatomic variability of developmental maturation of neuroanatomic structures in both typically and atypically developing populations can be discussed.

MRDD Research Reviews 2003;9:155-160 ๑ 2003 Wiley-Liss, Inc.

Key Words: magnetic resonance imaging; volume; quantitative brain anatomy; database; image analysis; morphometry

\section{INTRODUCTION}

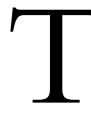
he neuroinformatics landscape in which human brain morphometry occurs has advanced dramatically over the past few years [Ascoli et al., 2003]. Rapid advancement in image acquisition methods, image analysis tools and interpretation of morphometric results make the study of in vivo anatomic analysis both challenging and rewarding. Magnetic resonance imaging technologies have provided a wide range of tools for characterizing brain structure. These tools have begun to revolutionize the way we see the brain in development, aging and disease. Magnetic resonance (MR) imaging can be sensitive to many physical properties of the brain, and the anatomic interpretation and characterization of these features provide a vast array of potential metrics of brain structure. These range from the volumetric characterization of structure as well as characterization of the distribution of parametric observations within structures. The MR signal represents a spatial and temporal mapping of specific structural, functional and compositional characteristics of the brain [Belliveau et al., 1991; Jenkins et al., 1999; Kwong et al., 1992; Makris et al., 1997; Rosen et al., 1993]. MR-based imaging methods are playing an increasingly more important role in the advance of the cognitive and clinical neurosciences [see Davidson et al., 2003; Durston, 2003; Eigsti and Shapiro, 2003 this issue; also see Casey, 2002; Casey et al., 2000; 2002; Casey and Munakata, 2002].
With respect to quantitative brain morphometry, the literature has been dominated by first volumetric [Kennedy et al., 1989], and subsequently by surface-based [Dale et al., 1999; Fischl et al., 1999a; 1999b; Thompson and Toga, 1996; Thompson et al., 2000] representations of structures, and their changes with time and disease. These different perspectives offer a complementary view of different organizational principles of brain structure. For example, the surface representation of the cerebral cortex is the natural spatial mapping dimension for functional processing in this structure. Cortical thickness along this surface offers information into the columnar dimension of cortical organization, whereas volumetric representation of regions of cortex may reflect the local tradeoffs of the global constraints on neural proliferation, cell density and number of neurons [Caviness et al., 1996; 1999].

As the literature expands, informatics tools for better interrogation of the literature as a whole are being developed. Databasing of the literature 'retrospectively' is critical to understanding the wealth of information that already exists about brain structure. However, this retrospective approach is relatively 'unconstrained' in terms of many details regarding data acquisition, image analysis, and subject characterization. Concurrently, many efforts are ongoing to create prospectively controlled studies, facilitating multi-site collaboration in order to capitalize on the synergy represented by the widespread study of many common neurological and psychiatric disorders. The prospective approach can be expected to overcome many of the inherent vagaries that inhabit retrospective interpretation of the literature. But concerted community consensus is required, and many years of prospective acquisition and analysis are required before the vastness of the existing literature can be approximated. Clearly, both of these types of approach can play a valuable role in the analysis of brain structures.

In the remainder of this paper, we will briefly review some of the issues regarding the anatomic analysis of MR

Grant sponsor: Human Brain Project; Grant numbers: NS34189, NS20489, NS02126; Grant sponsors: the Don and Linda Carter Foundation, the Fairway Trust, and the Giovanni Armenise - Harvard Foundation for Advanced Scientific Research.

*Correspondence to: David N. Kennedy, Ph.D., MGH-CMA, $14913^{\text {th }}$ Street,

Charlestown, MA 02129. E-mail: dave@cma.mgh.harvard.edu

Published online in Wiley InterScience (www.interscience.wiley.com).

DOI: $10.1002 / \mathrm{mrdd} .10075$ 
images, and tour some sample results from a prototype retrospective database of neuroanatomic volumetric information.

\section{METHODS}

Anatomic structures can be quantitatively characterized by morphometric descriptors with respect to both their volumetric and surface representations. Common descriptors include: size (volume, surface area, etc.) [Fischl and Dale, 2000; Fischl et al., 1999a; Kennedy et al., 1989], shape (parameterization, deformation, etc.) [Davatzikos and Bryan, 1996; DeQuardo et al., 1999; Free et al., 2001; Hogan et al., 2000; Kovacs et al., 1998; McInerney and Terzopoulos, 1996; Thompson and Toga, 1996], anatomic location (relative to a specific coordinate system) [Fischl et al., 1999a; 1999b; Talairach et al., 1967; Talairach and Tournoux, 1988; Thompson et al., 1997], and composition characteristics (parameter distributions) [Rosas et al., 2002]. See [Kennedy et al., 2002] for a more comprehensive exposition on these classes of descriptors.

Each of these classes of observation requires the identification of the anatomic structure as an initial step. This 'segmentation' of an image into anatomic structures can be performed to identify the border of the structure, or identification of all voxels contained within the structure. Segmentation has a long and rich literature, the reader is directed to comprehensive reviews such as [Clarke et al., 1995; Viergever et al., 2001] for a more detailed presentation on this topic. Another approach receiving substantial use is 'voxel-based morphometry' (VBM) [Ashburner and Friston, 2000]. In summary, images are subjected to a spatial normalization and pixel classification procedure (to define voxels containing gray matter, white matter and CSF, for example) and then a voxel-wise statistic on these classifications is performed [e.g., Sowell et al., 1999]. This statistical test can be for comparison between subject groups (i.e., disease vs. normal) or between classification and an extrinsic parameter (i.e., age). The spatial and statistical issues in this class of analysis are analogous to statistical parametric mapping of functional activation analysis [Friston et al., 1995] and this methodological overlap can be used to practical advantage. However, despite its ease of use, the spatial smoothing and inter-subject registration issues that are raised result in a decrease of effective anatomic and spatial resolution and spatial variabil- ity in sensitivity [Ashburner and Friston, 2001; Bookstein, 2001].

A general reference for image analysis tools, including those useful for segmentation and morphometric analysis, is the Internet Analysis Tools Registry (IATR - http://www.cma.mgh.harvard. edu/iatr). The IATR is specifically designed to facilitate tool sharing, both for the developer and consumer of tools. In addition to the tools themselves, the results of tool use (i.e., morphometric data) also needs methods for enhanced information sharing. While individual investigators or groups of investigators can acquire and analyze a substantial amount of data, it is also likely that many of the problems facing neuroscientists will require more data resources than can be acquired at an individual site. To this end, an Internet Brain Volume Database (IBVD - http://www.cma.mgh.harvard. edu/ibvd/) has been created to reposit volumetric neuroanatomic data. This resource is primarily concerned with published group and individual volumetric results. For each publication indexed, the reported groups and individuals are characterized with respect to the demographic, clinical and anatomic measurements reported. To facilitate interdatabase federation, many elements of the database are related to 'concept' entries in the Unified Medical Language System (UMLS) [Hole \& Srinivasan, 2003] including the anatomic nomenclature (through NeuroNames [Bowden and Martin, 1995; Bowden and Dubach, 2003]] and diagnosis codes (DSM-4 and ICD-10).

Initial prototype development has been performed for the creation of the IBVD. For feasibility testing, the database has been initially created and tested using data obtained from the many studies already performed to date in our laboratory and a sampling of data derived from the published literature. The database supports results from both groups of individuals and individuals themselves. Specifically as of this writing, 80 publications, encompassing 209 subject groups, 1127 volumetric observations in these groups, spanning 25 clinical diagnostic criteria are entered. In addition, 1660 individuals have been entered for which there are 7323 individual structural volume entries. These volume measurements cover a wide range of anatomic structures (37 discrete structures), from total brain volume, to subcortical nuclei. Extensions to include different species, as well as post mortem data have been implemented.

\section{RESULTS}

As introduced above, the MR scan must be segmented as an antecedent step for any subsequent analysis of morphometric properties. Figure 1 shows an example of the borders generated for cortical exterior and cortex-white mater using the tools described in [Dale et al., 1999]. The results of segmentation can be quantified in terms of volume and surface properties, as well as providing the necessary information for use in various surface and volume rendering schemes.

Independent of acquisition or analysis method, the literature has amassed a wealth of quantitative volumetric results over the past decade. The IBVD provides a mechanism by which to accumulate and inter-relate these observations across site, methods, study populations, etc. It must be noted that, as a relatively new database, only a small portion of the vast volumetric literature is indexed as of this writing (see Methods section). However, as a proof of concept, a number of interesting observations can be gleaned from this implementation. Figure 2a shows the result of a query for 'total brain volume in normal subjects' plotted as a function of age. This result is generated from 27 subject groups reported in 10 papers. The normal developmental trajectory is relatively apparent despite mixed acquisition and analysis methods. Figure $2 \mathrm{~b}$ demonstrates a similar query where various disorders are included in contrast to the normative populations. It can be seen, however, that most disorders, relative to the population and methodological variances, do not make a substantial deviation from the normal trajectory on this gross large scale volumetric representation.

Refining these types of literaturebased observations in anatomic scale, Figure 3 demonstrates the results of an IBVD query for the partitioning of the cerebrum in terms of cortex and white matter as a function of age in the normal population (30 groups reported in 10 publications). The relative size differences between these anatomic compartments can be appreciated, as well as an essence of the differing age relationship of these anatomic compartments.

We can probe even deeper into the anatomic hierarchy of the cerebrum to look at total caudate nucleus volume in both normal and pathologic populations as shown in Figure 4. Here again, we see the emergence of a developmental trajectory which is relatively independent of acquisition and analysis method, and that many of the reported pathologic condi- 


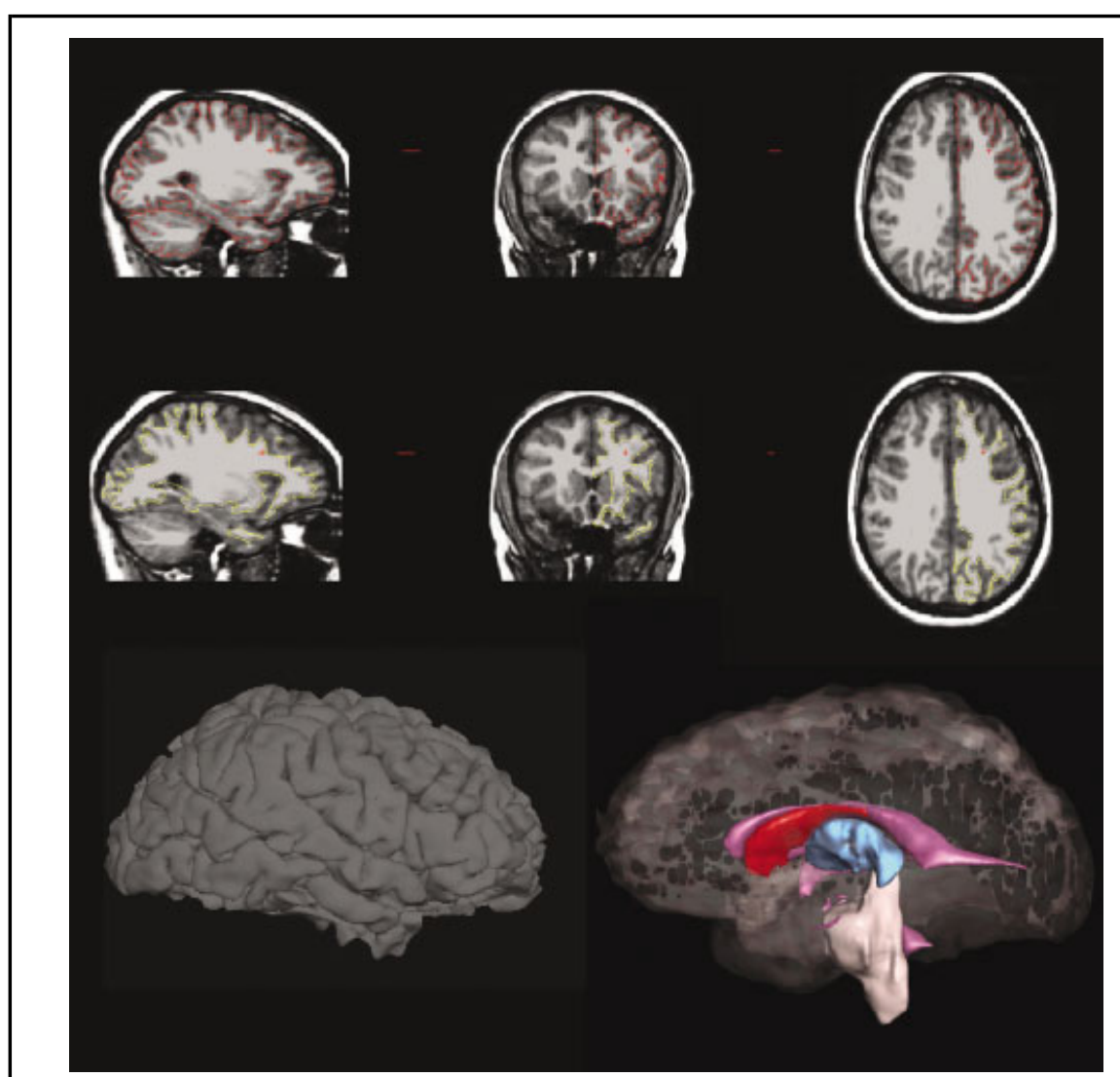

Fig. 1. Example segmentation for volume and surface analysis. The cerebral exterior border is demonstrated in the principle views in the top row. The cerebral cortex - cerebral white matter border is demonstrated in the principle views in the middle row. The bottom row demonstrates the surface and volume rendering visualization of the results of anatomic segmentation.

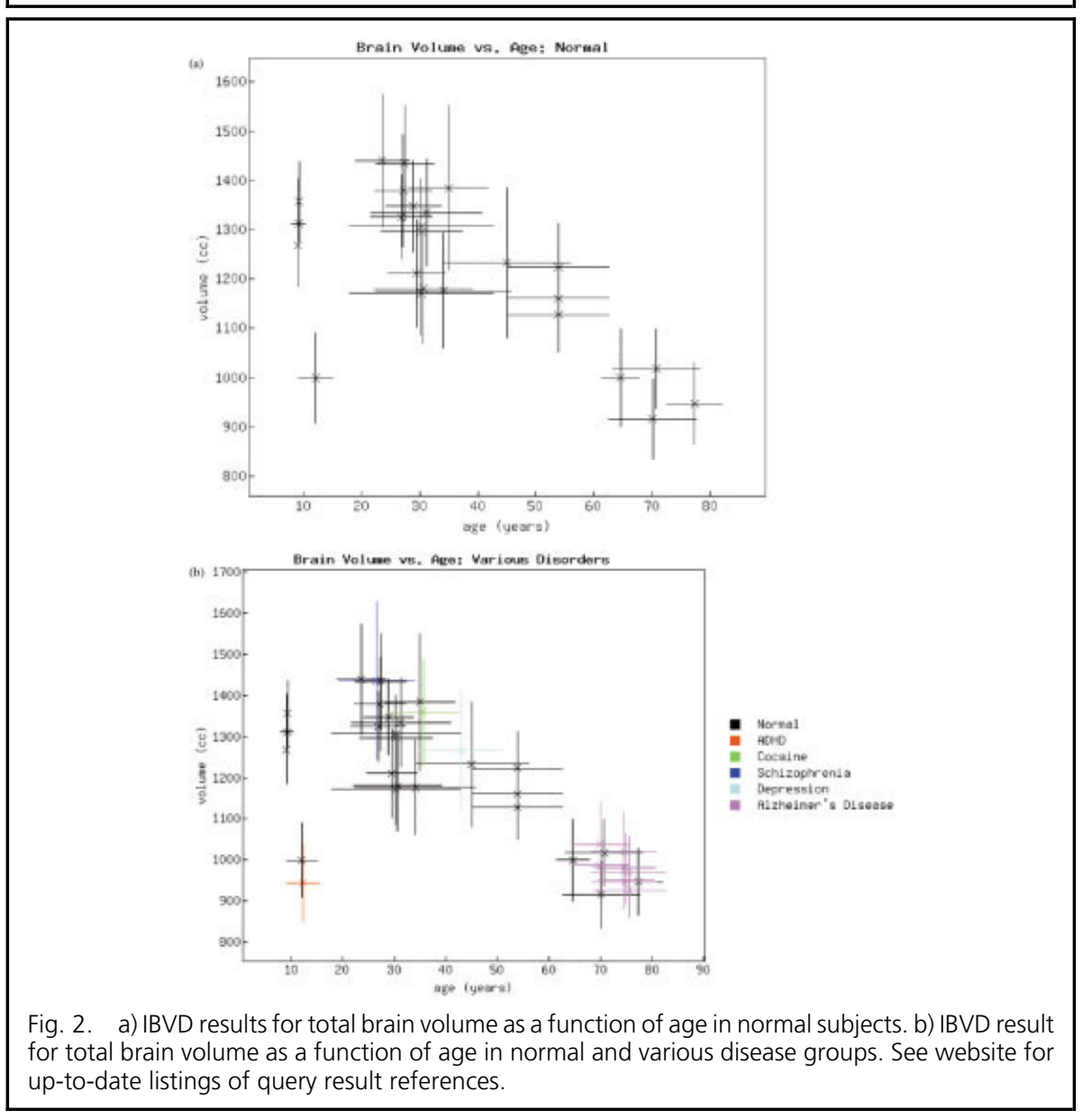

tions have a profound effect on this structure volume (see particularly the Huntington's Disease caudate volumes at the older age range).

\section{DISCUSSION AND CONCLUSIONS}

The expanding literature on the results of morphometric analysis poses a daunting task for scientists interested in the expression of neuroanatomy in vivo. The wealth of data, the breadth of classes of surface and volume based observations, and the expansive range of normal age and disease related populations that have been studied clearly highlight the need for more advanced neuroinformatics tools to catalogue and navigate this information. As tools for image analysis are developed, these need to be shared with potential tool users. As results are generated, these need to be electronically accessible and searchable. Two such resources, the Internet Analysis Tools Registry (IATR) and Internet Brain Volume Database (IBVD) introduced above are examples of such possible informatics tools.

Historically, many morphometric characterizations have been found to be sensitive to subtle structural differences in the brain between subjects with neurological and psychiatric disorders compared to normal subjects. As the developmental trajectory of the normal brain, as well as its response to pathologic insult, is a 'moving' target, complex problems in interpretation is imposed by the ubiquity of cross-sectional experimental designs. Every morphometric parameter by which the brain might be described is dependent upon developmental stage and undergoes massive transformation from conception to old age. Ideally, the morphometric characterization of the brain would span the entire lifespan and be conducted longitudinally in individual subjects. While this overall goal may be impractical to accomplish at the moment, various prospective longitudinal efforts are underway. Within the methodological analysis of specific research groups, an initial glimpse of the form of the normal developmental trajectory for some anatomic structures can begin to be formed [Chung et al., 2001; Giedd et al., 1996; Giedd et al., 1999; Paus et al., 1999; Thompson et al., 2000]. Such valuable studies of their own right can easily be anticipated to be highly magnified in their significance when performed as a comprehensive, multi-site, longitudinal study utilizing a homogeneous analysis method. 

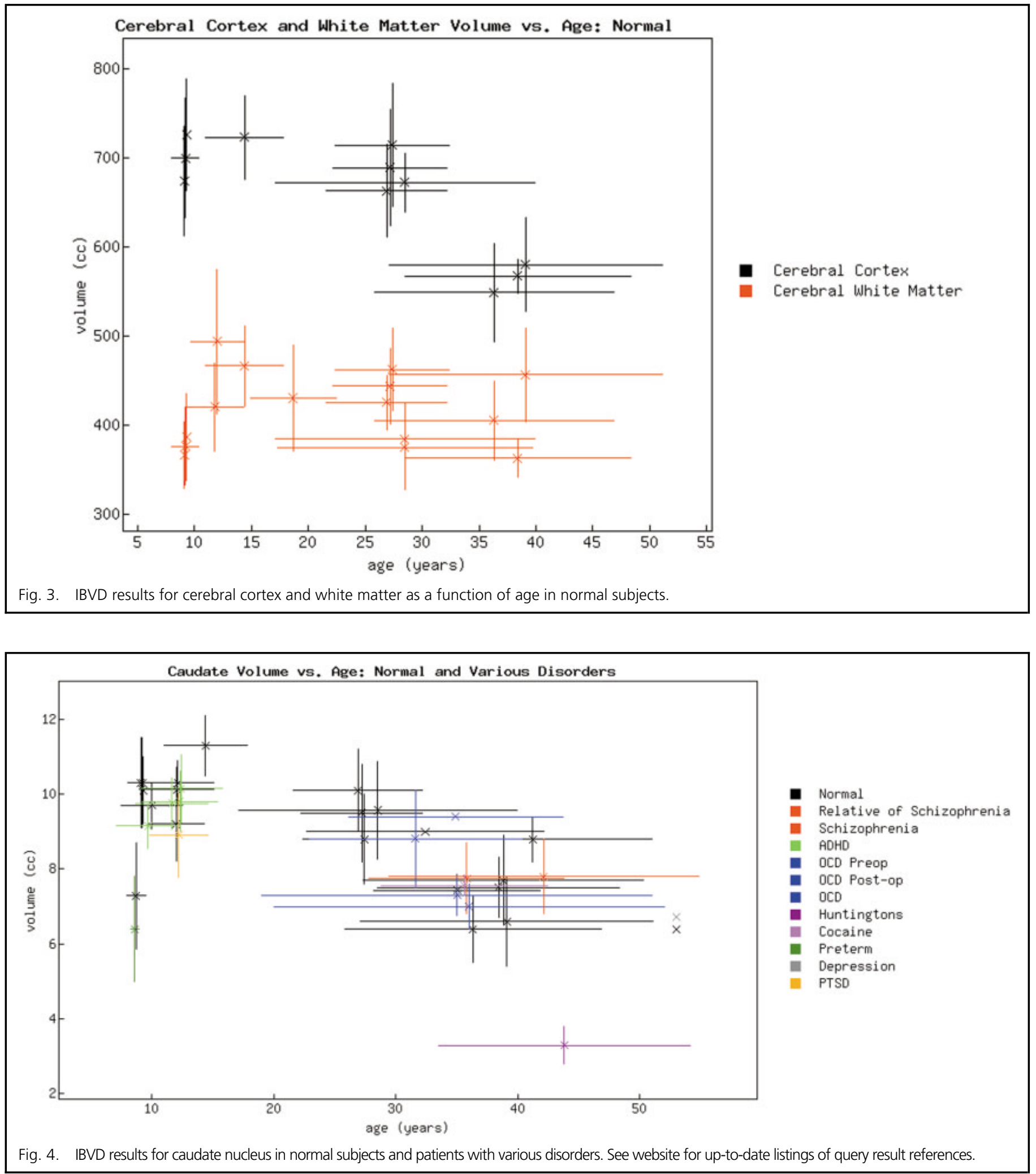

As retrospective, 'uncontrolled' databasing of volumetric data proceeds, important issues are raised regarding the interpretation of these data. One cannot escape acknowledgement of the utility of studies that have a large $(\mathrm{N}>300)$ number of subjects. Many of the anatomic differences to be found in morphometric studies can be expected to be quite subtle. Large subject pools, by necessity, place large demands on the level automation necessary in the image analysis methods. A number of systems with very high levels of automation have been introduced [for example, Dale et al., 1999; Kikinis et al., 1999; Rosas et al., 2002;
Watkins et al., 2001]. Calibration studies for these systems have been performed that indicate a high degree of volumetric similarity for results generated with these automated systems in comparison to manual segmentation [Rosas et al., 2002]. It is also clear, however, that despite the nature of the errors introduced 
by human segmentation, a different set of errors can be introduced by the automation process, particularly when evaluated on a voxel-decision by voxel-decision basis. In other words, automation can not eliminate the need for human review and potential final editing, which adds analysis time even to a completely automated initial analysis system. However, the overall time savings can be substantial, and the relative systematization of automated versus manual segmentation systems make these kinds of technological advances critical for the pursuit of the morphometric profile of neuroanatomic structures.

The literature and the demonstrations in the results sections indicate that different anatomic structures have different temporal profiles of development. The temporal profile of relatively large scale structures such as total brain volume can mask the detailed and varied profiles that will be followed by their component small-scale structures. It can also be appreciated that dramatic changes (expansion) occur during the early phase of brain growth from conception to childhood, and that a massive repartitioning occurs in the years of adolescence when this general rapid expansion converts to a slow contraction through the later years in life. Yet each set of anatomic structures has a unique set of expansion and contraction rates and details of temporal timing that can be potentially anticipated in cross-sectional study designs, but can only be confirmed and accurately tracked in longitudinally-designed follow-up studies [Casey et al., 2000; Giedd et al., 1999].

As the wealth of morphometric information expands, an expanding emphasis is placed on the tools necessary to fully understand and integrate these data. These tools, in return, place emphasis on the needs and criteria for data integration. Data integration can be explored through sharing of common data with various tools, through standardization of acquisition protocols across sites, and pooling of multi-site data in order to accumulate studies with substantially larger numbers of subjects than are currently feasible. Through such examples of federated and integrated neuroinformatics resources, progress towards delivery of a comprehensive infrastructure to the neuroscience community is being made.

\section{ACKNOWLEDGEMENTS}

This work was supported in part by grants NS34189 as part of the Human
Brain Project, NS20489, NS02126, the Don and Linda Carter Foundation, the Fairway Trust, and the Giovanni Armenise - Harvard Foundation for Advanced Scientific Research.

\section{REFERENCES}

Ascoli GA, De Shutter E, Kennedy DN. 2003. An information science infrastructure for neuroscience. Neuroinformatics1:1-2.

Ashburner J, Friston KJ. 2000. Voxel-based morphometry - the methods. Neuroimage 11: 805-821.

Ashburner J, Friston KJ. 2001. Why voxel-based morphometry should be used. Neuroimage 14: $1238-1243$.

Belliveau JW, Kennedy DN, McKinstry RC, et al. 1991. Functional mapping of the human visual cortex by magnetic resonance imaging. Science 254:716-719.

Bookstein FL. 2001. "Voxel-based morphometry" should not be used with imperfectly registered images. Neuroimage 14:1454-1462.

Bowden DM, Dubach MF. 2003. NeuroNames 2002. Neuroinformatics 1:43-60.

Bowden D, Martin R. 1995. NeuroNames brain hierarchy. Neuroimage 2:63-83.

Casey BJ. 2002. Neuroscience. Windows into the human brain. Science, 296:1408-1409.

Casey BJ, Munakata Y. 2002. Converging methods in developmental science: an introduction. Dev Psychobiol 40:197-199.

Casey B, Giedd J, Thomas K. 2000. Structural and functional brain development and its relation to cognitive development. Biol Psychol 54:241-257.

Casey BJ, Tottenham N, Fossella J. 2002. Clinical, imaging, lesion, and genetic approaches toward a model of cognitive control. Dev Psychobiol 40:237-254.

Caviness VS, Kennedy DN, Bates J, et al. 1996. The developing human brain: A morphometric profile. In: Thatcher RW, Lyon GR, Rumsey J, et al., eds. Developmental neuroimaging: mapping the development of brain and behavior. pp. 3-14. New York: Academic Press.

Caviness VS, Lange NT, Makris N, et al. 1999. MRI-Based brain volumetrics: Emergence of a developmental brain science. Brain and Development 21:289-295.

Chung M, Worsley K, Paus T, et al. 2001. A unified statistical approach to deformation-based morphometry. Neuroimage 14:595-606.

Clarke L, Velthuizen R, Camacho M, et al. 1995 MRI segmentation: methods and applications. Magn Reson Imaging 13:343-368.

Dale A, Fischl B, Sereno M. 1999. Cortical surface-based analysis. I. Segmentation and surface reconstruction. Neuroimage 9:179-194.

Davatzikos C, Bryan RN. 1996. Using a deformable surface model to obtain a shape representation of the cortex. IEEE Transactions on Medical Imaging 15:785-795.

Davidson MC, Thomas KM, Casey BJ. 2003. Imaging the developing brain with $\mathrm{fMRI}$. Ment Retard Dev Disabil Res Rev 9:161-167.

DeQuardo J, Keshavan M, Bookstein F, et al. 1999. Landmark-based morphometric analysis of first-episode schizophrenia. Biol Psychiatry 45:1321-1328

Durston S. 2003. A Review of the Biological Bases of ADHD: What have we learned from imaging studies? Ment Retard Dev Disabil Res Rev 9:184-195.

Eigsti I-M, Shapiro T. 2003 A systems neuroscience approach to autism: Biological, cognitive and clinical perspectives. Ment Retard Dev Disabil Res Rev 9:206-216.

Fischl B, Dale A. 2000. Measuring the thickness of the human cerebral cortex from magnetic res- onance images. Proc Nat Acad Sci USA 97: 11050-11055

Fischl B, Sereno M, Dale A. 1999a. Cortical surface-based analysis. II: Inflation, flattening, and a surface-based coordinate system. Neuroimage 9:195-207.

Fischl B, Sereno M, Tootell R, et al. 1999b. Highresolution intersubject averaging and a coordinate system for the cortical surface. Human Brain Mapping 8:272-284.

Free S, O'Higgins P, Maudgil D, et al. 2001. Landmark-based morphometrics of the normal adult brain using MRI. Neuroimage 13:801-813.

Friston K, Holmes A, Worsley K, et al. 1995. Statistical Parametric Maps in functional imaging: A general linear approach. Human Brain Mapping 2:189-210.

Giedd JN, Snell JW, Lange N, et al. 1996. Quantitative magnetic resonance imaging of human brain development: ages 4-18. Cerebral Cort 6:551-560.

Giedd J, Blumenthal J, Jeffries N, et al. 1999. Brain development during childhood and adolescence: A longitudinal MRI study. Nat Neurosci 2:861-863.

Hogan R, Bucholz R, Choudhuri I, et al. 2000. Shape analysis of hippocampal surface structure in patients with unilateral mesial temporal sclerosis. J Digit Imaging 13:3942.

Hole WT, Srinivasan S. 2003. Adding NeuroNames to the UMLS Metathesaurus. Neuroinformatics 1:61-64.

Jenkins BG, Chen YI, Makris N, et al. 1999. An integrated strategy for evaluation of metabolic and oxidative defects in neurodegenerative illness using magnetic resonance techniques. Ann NY Acad Sci 893:214-242.

Kennedy DN, Filipek PA, Caviness VS. 1989. Anatomic segmentation and volumetric calcuations in nuclear magnetic resonance imaging. IEEE Trans Med Img 8:1-7.

Kennedy DN, Makris N, Herbert MR, et al. 2002. Basic principles of MRI and morphometry studies of human brain development. Developmental Science 53:268-278.

Kikinis R, Guttmann C, Metcalf D, et al. 1999. Quantitative follow-up of patients with multiple sclerosis using MRI: technical aspects. Journal of Magnetic Resonance Imaging 9: 519-530.

Kovacs I, Feher A, Julesz B. 1998. Medial-point description of shape: a representation for action coding and its psychophysical correlates. Vision Res 38:2323-2333.

Kwong KK, Belliveau JW, Chesler DA, et al. 1992. Dynamic magnetic resonance imaging of human brain activity during primary sensory stimulation. Proc Natl Acad Sci USA 89: 5675-5679.

Makris N, Worth AJ, Sorensen AG, et al. 1997. Morphometry of in vivo human white matter association pathways with diffusion-weighted magnetic resonance imaging. Annals of Neurology 42:951-962.

McInerney T, Terzopoulos D. 1996. Deformable models in medical image analysis: A survey. Med Image Anal 1:91-108.

Paus T, Zijdenbos A, Worsley K, et al. 1999. Structural maturation of neural pathways in children and adolescents: in vivo study. Science, 283:1908-1911.

Rosas HD, Liu AK, Hersch S, et al. 2002. Regional and progressive thinning of the cortical ribbon in Huntington's disease. Neurology 58:695-701.

Rosen B, Aronen H, Kwong K, et al. 1993. Advances in clinical neuroimaging: Functional MR imaging techniques. Radiographics 13:889-896. 
Sowell ER, Thompson PM, Holmes CJ, et al. 1999. In vivo evidence for post-adolescent brain maturation in frontal and striatal regions. Nature Neuroscience 2:859-861.

Talairach J, Tournoux P. 1988. Co-Planar Stereotaxic Atlas of the Human Brain. New York: Thieme Medical Publishers, Inc.

Talairach J, Szikla G, Tournoux P. 1967. Atlas d'Anatomie Stereotaxique du Telencephale: Paris: Masson.
Thompson P, Toga AW. 1996. A surface-based technique for warping three-dimensional images of the brain. IEEE Transactions on Medical Imaging 15:402-417.

Thompson PM, MacDonald D, Mega MS, et al. 1997. Detection and mapping of abnormal brain structure with a probabilistic atlas of cortical surfaces. Journal of Computer Assisted Tomography 21:567-581.

Thompson PM, Giedd JN, Woods RP, et al. 2000. Growth patterns in the developing brain de- tected by using continuum mechanical tensor maps. Nature 404:190-193.

Viergever M, Maintz J, Niessen W, et al. 2001. Registration, segmentation, and visualization of multimodal brain images. Comput Med Imaging Graph 25:147-151.

Watkins K, Paus T, Lerch J, et al. 2001. Structural asymmetries in the human brain: a voxelbased statistical analysis of 142 MRI scans. Cereb Cortex 11:868-877. 